\title{
On cylindrical near-field scanning techniques
}

\section{Appel-Hansen, Jørgen}

\section{Published in:}

I E E E Transactions on Antennas and Propagation

Publication date:

1980

\section{Document Version}

Publisher's PDF, also known as Version of record

Link back to DTU Orbit

Citation (APA):

Appel-Hansen, J. (1980). On cylindrical near-field scanning techniques. I E E E Transactions on Antennas and Propagation, 28(2), 231-234.

\section{General rights}

Copyright and moral rights for the publications made accessible in the public portal are retained by the authors and/or other copyright owners and it is a condition of accessing publications that users recognise and abide by the legal requirements associated with these rights.

- Users may download and print one copy of any publication from the public portal for the purpose of private study or research.

- You may not further distribute the material or use it for any profit-making activity or commercial gain

- You may freely distribute the URL identifying the publication in the public portal

If you believe that this document breaches copyright please contact us providing details, and we will remove access to the work immediately and investigate your claim 


\section{Communications}

\author{
On Cylindrical Near-Field Scanning Techniques
}

\author{
JøRGEN APPEL-HANSEN, MEMBER, IEEE
}

\begin{abstract}
The agreement between the coupling equations obtained in the literature by using the reciprocity theorem and the scattering matrix formulation is demonstrated. The field is expanded in cylindrical vector wave functions and the addition theorem for these functions is used. The communication may serve as a tutorial introduction to the cylindrical scanning techniques.
\end{abstract}

\section{INTRODUCTION}

With the use of expensive satellite communication links there is an increasing demand for accurate antenna measurements. One technique is near-field measurements where the field of the test antenna is probed on a surface close to the antenna. The far field is then obtained by Fourier inversion of the measured data. The types of surfaces used in practice are the planar, cylindrical, and spherical surfaces. The advantages and limitations of using the different surfaces are discussed in [1]. The present communication deals with the cylindrical scanning technique whereby the test antenna may simply be rotated in azimuth and the probe moved in steps along a vertical line.

Three major approaches have been used to transform the measured near-field data to computed far-field characteristics. In the first approach Brown and Jull [2] and Leach and Paris [3] enclosed the test antenna and the probe in proper surfaces so that a source free volume was obtained. Then, using the Lorentz reciprocity theorem for the source free volume, the coupling equation was derived. This expresses the output voltage of a detector connected to the probe. For brevity we shall refer to this approach as the Lorentz reciprocity theorem formulation. In the second approach, which we call the scattering matrix formulation, different types of scattering matrices can be used to derive the coupling equation [4]. The matrices relate the amplitudes of waveguide modes and expansion coefficients by linear matrix transformations. These can be taken as definitions or derived from Maxwell's equations. Two types of scattering matrices used in the derivation of the coupling equation are described below. Recently, in a third approach Borgiotti [5] presented an integral formulation which used a superposition of plane waves.

Of course, all three approaches give the same final result, but this cannot be seen directly from the above references due to the different approaches and notations. This communication was therefore initiated with the aim of demonstrating the agreement between the results obtained by the Lorentz reciprocity theorem formulation as presented in [3] and the scattering matrix formulation. Two differences in these approaches should be noted. The first difference is related to reciprocity and the manner in which the trans-

Manuscript received April 5, 1979; revised September 28, 1979.

The author is with the Electromagnetics Institute, Technical University of Denmark, DK-2800 Lyngby, Denmark. mitting and receiving characteristics of the antennas enter in the coupling equation. In the Lorentz reciprocity theorem formulation the transmitting characteristics of both antennas enter and reciprocity is assumed. In the scattering matrix formulation it is convenient to use the transmitting characteristic of the transmitting antenna and the receiving characteristic of the receiving antenna, but reciprocity is not assumed. Thus the scattering matrix formulation can be used for nonreciprocal antennas. An extended analysis related to reciprocity can be carried out by using the concept of the adjoint antenna [4], [6]. In order to show the agreement between the two approaches, the present communication limits itself by assuming reciprocity. The second difference is related to scattering. In the Lorentz reciprocity theorem formulation multiple scattering between the test and probe antennas is neglected, but scattering or reradiation is taken into account in the beginning of the derivation. However, during the steps of derivation reradiation is neglected in some integrals and gives no contribution in other integrals; see [3] for further details. In the scattering matrix formulation, reradiation and multiple scattering are neglected.

In the literature two types of scattering matrices have been used. Yaghjian [4] introduced the source scattering matrix. This type of matrix uses expansion coefficients related to modes based on the Hankel functions of the second kind and Bessel functions. With the time factor $e^{j \omega t}$, as is used here, the Hankel function expansion is applied for the radiated field and the Bessel function expansion is used for the incident field. In [7] and [8] the normal scattering matrix is used in spherical scanning techniques. This type of matrix is used in waveguide theory [6] and may also be used in the case of cylindrical scanning. It relates the amplitudes of the waveguide modes with the amplitudes of propagating incoming and outgoing modes in the space surrounding the antenna. Thus the received signal depends only upon propagating incoming modes. This might also seem most logical. However, using coordinate systems and transformations as described in this communication, the incident field is expressed by using an expansion of Bessel functions which represent standing waves. In order to find the amplitudes of propagating incoming modes, use is made of the fact that the Bessel functions can be expressed by a sum of Hankel functions of the first and second kind divided by two. In this manner the amplitudes used in the normal scattering matrix formulation become onehalf of the amplitudes used in the source scattering matrix formulation. Since the received signal must be independent of the type of matrix, the receiving characteristics in the source scattering matrix formulation become half of the receiving characteristics in the normal scattering matrix formulation. From the discussion given above it is expected that both scattering matrix formulations should be applicable. However, the author is aware of the fact that the discussion may not be complete, and more rigorous comparison between the merits and deficiencies of the two types of scattering matrices is left for further research. In the communication the normal scattering matrix is adopted.

It should be mentioned that the probe characterization used here is not separated as in [4]. Such a separation may be 
an advantage but is not discussed here. Along with the recent works by Paris, Leach, and Joy [9] and Joy, Leach, Rodrique, and Paris [10], it is hoped that this communication could be considered as a tutorial introduction to cylindrical scanning and facilitate further studies. Additional references may be found in [9] and [10]

\section{COUPLING EQUATION}

\section{A. Geometrical Configuration}

In the cylindrical scanning technique the probe is moved on an imaginary circular cylinder enclosing the antenna under test. An outline of the transmission system setup for this scanning technique is shown in Fig. 1. In the derivation of the coupling equation the probe is operated as a transmitting antenna and the antenna under test as a receiving antenna. Only part of the cylindrical scanning surface is shown. The properties of the receiving antenna are described in an $x y z$ coordinate system with its origin 0 conveniently chosen in or close to the antenna structure. Alternatively, properties may be described in cylindrical $\rho \phi z$ - and spherical $r \phi \theta-c o-$ ordinate systems with the conventional orientation relative to the $x y z$-coordinate system. A point $0^{\prime}$ characterizing the position of the probe is designated by $\left(\rho_{0}, \phi_{0},{ }_{2}\right)$. The properties of the probe may be described in an $x^{\prime} y^{\prime} z^{\prime}$-coordinate system with its origin at $0^{\prime}$, its $z^{\prime}$ axis parallel to the $z$ axis, and the positive direction of its $x^{\prime}$ axis intersecting the $z$ axis. When the probe is scanned, $\rho_{0}$ is kept constant and $\phi_{0}$ and $z_{0}$ are varied. Thus for discrete values of $z_{0}$ the test antenna is rotated $360^{\circ}$ in azimuth. Whenever needed, the properties of the probe may also be described in conventional cylindrical $\rho^{\prime} \phi^{\prime} z^{\prime}$ - and spherical $r^{\prime} \phi^{\prime} \theta^{\prime}$-coordinate systems with their origins at $0^{\prime}$.

As in the planar case, the antennas are considered as two port transducers [6]. One port is located at a plane $S_{0}$ inside the waveguide feed. As will be apparent from the following discussion, the other port may be considered as a cylindrical surface placed around the antenna. The amplitudes of the incoming and outgoing simple waveguide modes at $S_{0}$ are $a_{0}$ and $b_{0}$, respectively. As seen above, $a_{n}{ }^{s}$ and $b_{n}{ }^{s}$ are the amplitudes of propagating incoming and outgoing cylindrical modes in which the electromagnetic field can be expanded. Primes are used to characterize quantities related to the probe.

\section{B. Expansion in Cylindrical Modes}

The electric field $\bar{E}^{\prime}\left(\vec{r}^{\prime}\right)$ radiated by the probe can be expressed by the expansion

$$
\vec{E}\left(\bar{r}^{\prime}\right)=\sum_{n=-\infty}^{\infty} \int_{-\infty}^{\infty}\left\{b_{n}{ }^{\prime} 1(\gamma) \bar{M}_{n \gamma}{ }^{(4)}\left(\bar{r}^{\prime}\right)+b_{n}{ }^{\prime 2}(\gamma) \bar{N}_{n \gamma}{ }^{(4)}\left(\bar{r}^{\prime}\right)\right\} d \gamma
$$

where $\vec{r}^{\prime}$ is the position vector characterizing the field point and $\vec{M}_{n \gamma}{ }^{(4)}\left(\vec{r}^{\prime}\right)$ and $\bar{N}_{n \gamma}{ }^{(4)}\left(\bar{r}^{\prime}\right)$ are elementary cylindrical vector wave functions derived from a cylinderical scalar function with azimuthal index $n$, propagation constant in the $z^{\prime}$ direction $\gamma$, and, as indicated by the superscript index (4), based on the Hankel functions of the second kind $H_{n}{ }^{(2)}\left(\kappa \rho^{\prime}\right)$ with $k=\sqrt{k^{2}-\gamma^{2}}$, where $k$ is the wavenumber of free space. For further details see [3], [4], and [11]. It is seen that the coefficients $b_{n}{ }^{\prime} s(\gamma)$, where $s=1,2$, are the weights or amplitudes with which, for given values of $n$ and $\gamma$, the elementary

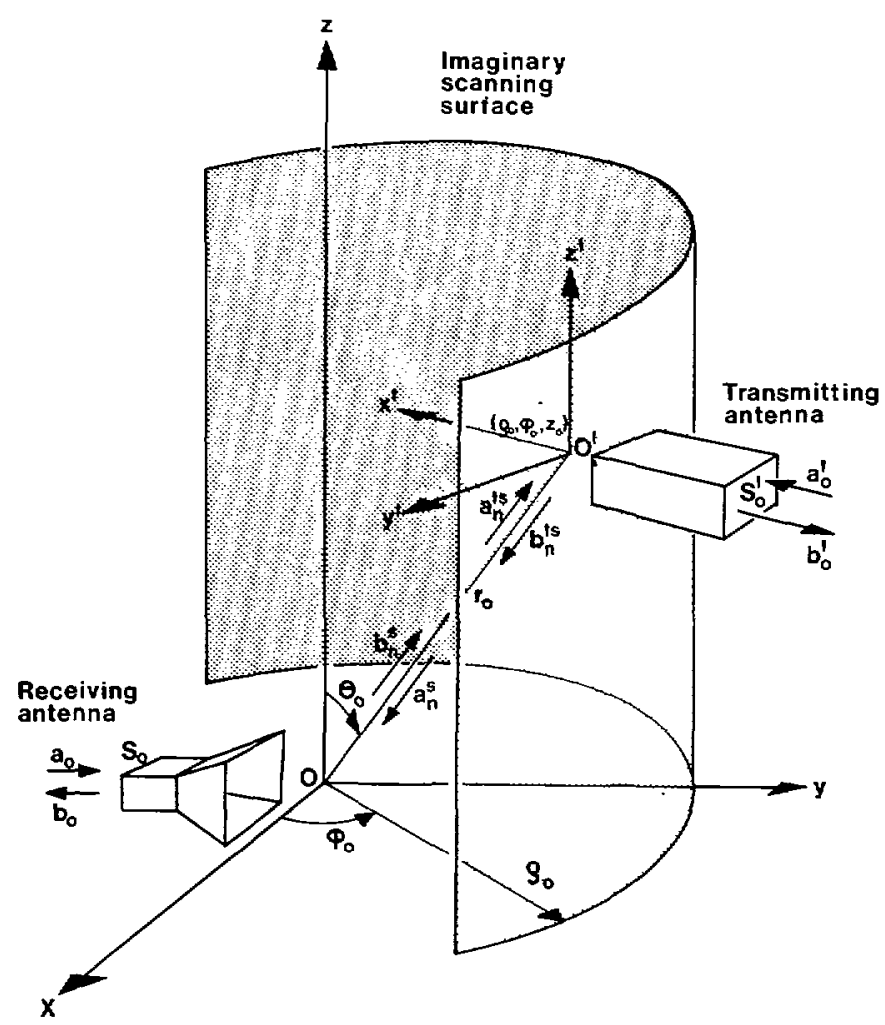

Fig. 1. Transmission system setup for cylindrical scanning.

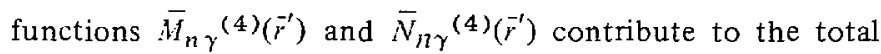
field. For this reason, and because the Hankel functions of the second kind represent outward propagating waves, we call $b_{n}{ }^{\prime} s(\gamma)$ spectrum density functions of propagating outgoing waves.

In order to derive the coupling equation it is desirable to express $\bar{E}^{\prime}\left(\bar{r}^{\prime}\right)$ in the $p \phi z$-coordinate system linked to the receiving test antenna. This is done by making use of the vector translation theorem for the cylindrical vector wave functions [3] (it is for easy reference to this form of the translation theorem that the probe is transmitting). The result is

$$
\begin{aligned}
\bar{E}(\bar{r})= & \sum_{n=-\infty}^{\infty} \int_{-\infty}^{\infty}\left\{b_{n}{ }^{1}(\gamma) \cdot \sum_{m=-\infty}^{\infty}(-1)^{m}\right. \\
& \cdot H_{n+m}{ }^{(2)}\left(\kappa \rho_{0}\right) e^{j m \phi_{0} e^{j \gamma z} 0 \bar{M}_{m \gamma}}{ }^{(1)}(\bar{r}) \\
& +b_{n}{ }^{\prime 2}(\gamma) \sum_{m=-\infty}^{\infty}(-1)^{m} H_{n+m}{ }^{(2)}\left(\kappa \rho_{0}\right) \\
& \left.\cdot e^{j m \phi_{0} e^{j \gamma z} 0 \bar{N}_{-m \gamma}{ }^{(1)}(\bar{r})}\right\} d \gamma
\end{aligned}
$$

where $\bar{M}_{-m \gamma}{ }^{(1)}(\bar{r})$ and $\bar{N}-m \gamma^{(1)}(\bar{r})$ are based on the Bessel functions $J_{-m}(\kappa \rho)$, as indicated by the superscript (1). By substituting $-m$ for $m$, changing the sequence of summation, interchanging $n$ with $m$, and using the relation between the Bessel functions and the Hankel functions of the first and second kind, it is found that the test antenna is placed in a field $\bar{E}_{i}^{\prime}(\bar{r})$ given by the following expansion in propagating 
incoming waves:

$$
\bar{E}_{i}^{\prime}(\bar{r})=\sum_{n=-\infty}^{\infty} \int_{-\infty}^{\infty}\left\{a_{n}{ }^{1}(\gamma) \bar{M}_{n \gamma}{ }^{(3)}(\bar{r})+a_{n}{ }^{2}(\gamma) \bar{N}_{n \gamma}{ }^{(3)}(\bar{r})\right\} d \gamma
$$

where

$$
a_{n}{ }^{s}(\gamma)=\frac{1}{2} \sum_{m=-\infty}^{\infty}(-1)^{n} H_{m-n}{ }^{(2)}\left(\kappa \rho_{0}\right) e^{-j n \phi} 0 e^{j \gamma z} 0 b_{m}{ }^{\prime} s(\gamma),
$$

and the superscript index (3) indicates that $\bar{M}_{n \gamma}{ }^{(3)}(\vec{r})$ and $\bar{N}_{n \gamma}{ }^{(3)}(\bar{r})$ are based on the Hankel functions of the first kind. Since these kinds of Hankel functions represent inward propagating waves, we call the coefficients $a_{n}(\gamma)$ spectrum density functions of the propagating incoming waves. It is the necessity of using the factor $\frac{1}{2}$ in (4) which is the crucial point in the discussion of the difference between the source and normal scattering matrix formulation given in the Introduction.

\section{Scattering Matrix Formulation}

The relationship between the wave amplitudes $a_{0}$ and $b_{0}$ in the waveguide feed and the spectrum density functions $a_{n}{ }^{s}(\gamma)$ and $b_{n}{ }^{s}(\gamma)$ in the space surrounding the antenna is given by the scattering matrix formulation

$$
\begin{aligned}
& b_{0}=\Gamma_{0} a_{0}+\sum_{n=-\infty}^{\infty} \int_{-\infty}^{\infty} \sum_{s=1}^{2} R_{n}{ }^{s}(\gamma) a_{n}{ }^{s}(\gamma) d \gamma, \\
& b_{n}{ }^{s}(\gamma)=T_{n}{ }^{s}(\gamma) a_{\circ}+\sum_{m=-\infty}^{\infty} \int_{-\infty}^{\infty} \sum_{q=1}^{2} S_{n m}{ }^{s q}(\gamma, \beta) a_{m}{ }^{q}(\beta) d \beta,
\end{aligned}
$$

where $\Gamma_{0}$ is the reflection coefficient of the antenna, and its receiving, transmitting, and scattering properties are characterized by the receiving spectrum $R_{n} s(\gamma)$, the transmission spectrum $T_{n}{ }^{s}(\gamma)$, and the scattering spectrum $S_{n m} s q(\gamma, \beta)$, respectively.

\section{Coupling Equation}

The coupling equation for the transmission system in Fig. 1 may now be found. Using the probe scattering matrix formulation, i.e., (5) and (6) with primes, the spectrum density functions of the fields leaving the probe are found to be

$$
b_{n}{ }^{\prime} s(\gamma)=T_{n}{ }^{\prime s}(\gamma) a_{0}{ }^{\prime}
$$

when reradiation and multiple scattering between the probe and test antenna are neglected, i.e., $a_{m}{ }^{\prime} q(\beta)=0$. Insertion of (7) into (4) gives

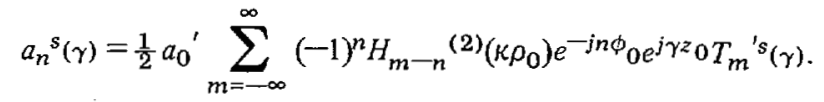

Let the test antenna be terminated with a load $Z_{l}$ with the reflection coefficient $\Gamma_{l}$, then $a_{0}$ and $b_{0}$ are related by

$$
a_{0}=\Gamma_{l} b_{0}
$$

Insertion of (8) and (9) into (5) gives

$$
b_{0}=\frac{a_{0}{ }^{\prime}}{2\left(1-\Gamma_{0} \Gamma_{l}\right)} \sum_{n=-\infty}^{\infty} e^{-i n \phi_{0}}
$$

$$
\begin{aligned}
& \times \int_{-\infty}^{\infty} \sum_{s=1}^{2}\left\{(-1)^{n} R_{n}{ }^{s}(\gamma) \sum_{m=-\infty}^{\infty} H_{m-n}{ }^{(2)}\left(\kappa \rho_{0}\right) T_{m}{ }^{\prime} s(\gamma)\right\} \\
& \cdot e^{j \gamma z} 0 d \gamma
\end{aligned}
$$

which is the coupling equation. This may be transformed to

$$
\begin{aligned}
b_{0}= & \frac{a_{0}{ }^{\prime}}{1-\Gamma_{0} \Gamma_{l}} \frac{4 \pi}{\eta_{0} Z_{0} k} \sum_{n=-\infty}^{\infty} e^{j n \phi_{0}} \\
& \times \int_{-\infty}^{\infty} \sum_{s=1}^{2}\left\{\kappa^{2} T_{n}^{s}(\gamma) \sum_{m=-\infty}^{\infty} H_{n+m}{ }^{(2)}\left(k \rho_{0}\right) T_{m}{ }^{\prime} s(-\gamma)\right\} \\
& \cdot e^{-i \gamma z} 0 d \gamma
\end{aligned}
$$

by making use of the reciprocity relation in [4, eq. (B 11a)] (corrected for a misprint and taking into account that the normal scattering matrix is used in the present paper)

$$
(-1)^{n} R_{-n}^{s}(\gamma)=\frac{8 \pi \kappa^{2}}{\eta_{0} Z_{0} k} T_{n}^{s}(-\gamma),
$$

where $\eta_{0}$ is the characteristic admittance of the propagating mode in the test antenna feedline and $Z_{0}$ is the free-space impedance.

It is now possible to observe the agreement between (11) and [3, eq. (23)]. In order to do so, [3, eq. (23)] has to be corrected for the fact that $\Lambda^{2}$ should be in the integrand, since $\Lambda^{2}=k^{2}-h^{2}$. Then agreement between (11) and [3, eq. (23)] appears, except for a normalization constant, by making the substitutions $b_{0}=v_{0}\left(r_{0}, \phi_{0}, z_{0}\right), \kappa=\Lambda$, $\gamma=h, \rho_{0}=r_{0}, T_{n}{ }^{1}(\gamma)=a_{n}(h), T_{n}{ }^{2}(\gamma)=b_{n}(h), T_{m}{ }^{1} 1(-\gamma)=$ $c_{m}(-h), T_{m}{ }^{\prime 2}(-\gamma)=d_{m}(-h)$. Thus it has been demonstrated that the scattering matrix formulation leads to the same result as obtained by using the Lorentz reciprocity theorem.

\section{INVERSION OF COUPLING EQUATION}

As in the case of planar scanning [12], it is convenient to introduce the symbol $D^{\prime}(n, \gamma)$ for a coupling product which we define by

$$
D^{\prime}(r, \gamma)=\sum_{s=1}^{2}\left\{\kappa^{2} T_{n}{ }^{s}(\gamma) \sum_{m=-\infty}^{\infty} H_{n+m}{ }^{(2)}\left(k \rho_{0}\right) T_{m}{ }^{\prime}(-\gamma)\right\}
$$

which is determined by the Fourier inversion of (11)

$$
\begin{aligned}
D^{\prime}(n, \gamma)= & \left(1-\Gamma_{0} \Gamma_{l}\right) \frac{\eta_{0} Z_{0} k}{16 \pi^{3} a_{0}{ }^{\prime}} \\
& \int_{-\infty}^{\infty} \int_{0}^{2 \pi} b_{0}\left(\phi_{0}, z_{0}\right) e^{-j n \phi_{0} e^{j \gamma z} 0} d \phi_{0} d z_{0} .
\end{aligned}
$$


For a known probe transmission spectrum $T_{m}{ }^{\prime}{ }^{\prime}(-\gamma)$, (14) provides one equation in the two unknowns $T_{n} s(\gamma)$ of the test antenna. Another equation may be obtained, e.g., by repeating the measurements for another aspect of the probe or with another known probe. After this, the solution of the two equations is straightforward. Cases where the determinant of the equations is zero may be solved as in the planar case [12].

In actual measurements $b_{0}\left(\phi_{0}, z_{0}\right)$ is sampled for the probe moved in a lattice with sample intervals $\Delta \phi_{0}$ and $\Delta z_{0}$ The angle $\phi_{0}$ is changed from 0 to $2 \pi$ during a revolution in azimuth. The $z_{0}$ traverse parallel to the $z$ axis has to be limited to the annular region in which radiation is appreciable. Thus final limits of $z_{0}$ can be introduced in the integral of (14). Then the coupling product $D^{\prime}(n, \gamma)$ can be written as a summation which is easily computed by using the fast Fourier transform algorithm. From band-limiting considerations it turns out that $\Delta \phi_{0}$ must be less than or equal to $\lambda / 2 a$, and $\Delta z_{0}$ must be less than or equal to $\lambda / 2$. Furthermore, practice seems to reveal that it is sufficient if the probe remains a few wavelengths away from the test antenna in order to avoid reactive near-field and multiple scattering effects. For further details, including the determination of probe characteristics from far-field probe data, see [3] and [4].

\section{ACKNOWLEDGMENT}

The author wishes to thank Kim Adamsen, Ernst Krogager, and Frank Jensen for reading and commenting on the manuscript. In particular, thanks are due to Flemming Holm Larsen for many fruitful discussions on the subject.

\section{REFERENCES}

[1] P. F. Wacker and A. C. Newell. "Advantages and disadvantages of planar, circular cylindrical, and spherical scanning and deseription of the NBS antenna scanning facilities." Antenna Testing Techniques. Workshop at ESTEC. European Space Agency. ESA SP 127, preprint. pp. 115-121. June 1977.

[2] J. Brown and E. V. Jull, "The prediction of aerial radiation patternis from near-field measurements." Inst. Elec. Eng.. Paper No. 3649 E. Nov. 1961.

[3] W. M. Leach. Jr.. and T. Paris. "Probe compensated near-field measurements on a cylinder," IEEE Trans. Antennas Propagat., vol. AP-21, pp. 435-445, July 1973.

[4] A. D. Yaghjian, "Near-fjeld antenna measurements on a cylindrical surface: A source scattering matrix formulation," Nat. Bur. Stand. NBS Tech. Note 696. pp. 1-32. July 1977.

[5] G. V. Borgiotti. "Integral equation formulation for probe cortected far-field reconstruction from measurements on a cylinder." IEEE Trans. Antennas Propagat., vol. AP-26, pp. 572-578, July 1978.

$[6]$ D. M. Kerns, "Plane-wave scattering-matrix theory of antennas and antenna-antenna interactions," Nat. Bur. Stand.. NBSIR 78-890. pp. I-277, June 1978.

[7] P. F. Wacker. "Non-planar near-field measurements: Spherical scanning." Electromag. Div.. Nat. Bur. Stand.. Boulder, CO. NBSIR 75-809, June 1975.

[8] F. Holm Larsen, "SNIFTC_-Spherical near-field transformation program with probe correction--Manual." Electromag. Inst.. Tech. Univ. Denmark. R 201, Oct. 1978.

[9] D. T. Paris. W. M. Leach. Jr.. and E. B. Joy. "Basic theory of probe-compensated near-field measurements." IEEE Trans. Antenna Propagat., vol. AP-26. pp. 373-378. May 1978.

[10] E. B. Joy, W. M. Leach, Jr., G. P. Rodrigue, and D. T. Paris. "Applications of probe-compensated near-field measurements." IEEE Trans. Antennas Propagat., vol. AP-26, pp. 379-389. May 1978.

[11] J. A. Stratton, Electromagnetic Theory. New York: McGraw-Hill. 1941 , pp. 360, 361, 392-395.

[12] D. M. Kerns. "Correction of near-field antenna measurements made with an arbitrary but known measuring antenna," Electron. Lett., vol. 6. pp. 346-347. May 1970.

\section{The Resolution Limit of a Variable Baseline Synthetic Aperture Antenna}

\section{N. EL-BEHERY AND R. H. MACPHIE, SENIOR MEMBER, IEEE}

\begin{abstract}
The resolution capabilities of a variable baseline correlation interferometer are analyzed using a maximum likelihood estimator algorithm for the outpuit data. Optimum estimates (in the Cramer-Rao sense) for two sources of both equal and unequal strengths, for different levels of receiver-background noise, and for finite integration (averaging) times are obtained. A likelihood probability $p_{n}(n=0,1,2,3, \cdots)$ is also obtained from the measured data, and the resolution limit is defined to be that for which the probabilities $p_{1}$ and $p_{2}$ are equal (on the average). It is shown by a computer simulation that the average resolution limit improves (decreases) with increased integration time, exceeding the traditional Rayleigh limit by an arbitrary amount even for quite unequal source strength ratios and large levels of receiver-background noise.
\end{abstract}

\section{INTRODUCTION}

The ability of an imaging system to resolve two closely spaced point sources has traditionally been measured by Lord Rayleigh's criterion of resolution [1], introduced a century ago in connection with prism and grating spectroscopes. This criterion, which deems two equal components to be resolved when the principal maximum of the intensity distribution of one source falls on the first minimum of the second source's distribution, is rather conservative and results in a 19-percent dip between the equal maxima of the combined distribution. Moreover, the criterion is silent in regard to components of unequal strength and on the problem of noise in the measurements.

In the past few decades a large number of radar engineers have addressed themselves to the problem of detecting single, double, and multiple targets (both coherent and incoherent) in the presence of noise. The task of detecting and estimating the unknown number of targets was simultaneously considered by Young [2] and Ksienski and McGhee [3]. In both works a decision-theoretic approach divided the problem into hypothesis testing and estimation using Bayes' method. Due to the difficulty of this formulation both approaches were limited to the two target case. Young calculated the one-two target estimator using $a$ priori knowledge about the target parameters but did not investigate either the processor accuracy or bias. Ksienski and McGhee concentrated on the estimation aspect for the two target case, a point which they considered before [4]. They showed by experimental implementation of the two target processor that it is possible to resolve two targets below a beamwidth separation at a 10-dB signal-to-noise ratio (SNR).

More recently, Steinberg [5] considered the resolution problem in synthetic aperture radar (the coherent case). Assuming additive two-dimensional Gaussian noise, he developed a probability function which is a measure of the dip of a prescribed amount $k \leqslant 1$ that exists between the peaks of the two received signals. His approach, therefore, is lineally descended from Rayleigh's in that a dip between the peaks plays an im-

Manuscript received July 9, 1979; revised October 19, 1979. This work was supported by the NSERC (Ottawa) under Grant No. A-2176.

I. N. El-Behery is with Ontario Hydro, Toronto, ON, M1C 1X6, Canada.

R. H. MacPhie is with the Electrical Engineering Department, University of Waterloo, Waterloo, ON, N2L 3G1, Canada. 